\title{
Adsorção dos corantes R016, RR2 e RR141 utilizando lodo residual da indústria têxtil
}

\section{Adsorption of dyes R016, RR2 and RR141 using residual sludge of textile industry}

\author{
Andressa Regina Vasques \\ Mestre em Engenheira Química pela Universidade Federal de Santa Catarina (UFSC). Doutoranda em Engenharia Química pela UFSC
}

\begin{abstract}
Selene M. A. Guelli U. de Souza
Doutora em Engenharia Mecânica pela UFSC. Professora Adjunto IV do Departamento de Engenharia Química e Engenharia de Alimentos da UFSC.

Orientadora e pesquisadora da UFCS
\end{abstract}

\section{Letícia Weissenberg}

Estudante de Graduação em Engenharia Química. Bolsista de Iniciação Científica pela Universidade Regional de Blumenau (FURB)

\begin{abstract}
Antônio Augusto Ulson de Souza
Doutor em Engenharia Mecânica pela UFSC. Professor Adjunto IV do Departamento de Engenharia Química e Engenharia de Alimentos da UFSC. Orientador e pesquisador da UFSC
\end{abstract}

José Alexandre Borges Valle

Doutor em Engenharia Mecânica pela UFSC. Professor Adjunto do Departamento de Engenharia Química da FURB. Orientador e pesquisador da FURB

\section{Resumo}

A adsorção é uma das técnicas empregadas com sucesso para remoção efetiva da cor presente em efluentes têxteis. Com o objetivo de avaliar os diferentes parâmetros adsortivos, bem como determinar a eficiência de um adsorvente alternativo desenvolvido a partir de lodo residual têxtil na remoção de corantes, foram determinadas curvas de cinética de adsorção e isotermas. Por meio dos dados cinéticos e de equilíbrio obtidos, verificou-se que a $25^{\circ} \mathrm{C}$ a adsorção foi favorável para todos os corantes, sendo esta a melhor condição para os corantes RO16 e RR2 na ausência de sais. Para o corante RR141, a adição de $\mathrm{NaCl}$ aumentou a capacidade de adsorção do adsorvente no equilíbrio e a adição de $\mathrm{Na}_{2} \mathrm{SO}_{4}$ favoreceu a adsorção para o corante RO16, ao contrário do que se observou para os outros dois corantes. A quantidade máxima de corante adsorvida por unidade de massa de adsorvente $\left(q_{\text {max }}\right)$ nas melhores condições adsortivas para os corantes R016, RR2 e RR141 foi de 81,30,53,48 e 78,74 mg.g ${ }^{-1}$, respectivamente.

Palavras-chave: adsorção; corantes; sais; indústria têxtil; lodo residual têxtil.

\section{Abstract}

The adsorption is one of the techniques that have been successfully used for effective removal of the dyes present in textile effluents. With the objective to evaluate the different adsorptive parameters, as well as determining the efficiency of one alternative adsorbent in the removal of dyes, kinetics and equilibrium data of adsorption were determined. By the kinetic data and of equilibrium, it was verified that the adsorption was favorable for all the dyes in $25^{\circ} \mathrm{C}$, being the best condition for the dye RO16 and RR2 in the total absence of salt. For the dye RR141, the addition of NaCl increased the adsorption capacity of adsorbent in the equilibrium and the addition of $\mathrm{Na}_{2} \mathrm{SO}_{4}$ favored the adsorption for the dye RO16, in contrast to what was observed for the two other dyes. The maximum quantity of dye adsorbed per unit mass of adsorbent $\left(q_{\max }\right)$ in the best adsorptive conditions for the dyes RO16, RR2 and RR141 was of $81.30,53.48$ and 78.74 $\mathrm{mg} \cdot \mathrm{g}^{-1}$ respectively.

Keywords: adsorption; dyes; salts; textile industry; residual textile sludge.

Endereço para correspondência: Andressa Regina Vasques - Programa de Pós-Graduação em Engenharia Química do Departamento de Engenharia Química e Engenharia de Alimentos da Universidade Federal de Santa Catarina, Campus Universitário Trindade - 88040-900, Florianópolis (SC), Brasil - Tel: (48) 3721-5231 E-mail: andressa vasques@hotmail.com

Recebido: 07/12/09 - Aceito: 27/07/11 - Reg. ABES: 17709 


\section{Introdução}

As indústrias têxteis têm grande dificuldade em tratar eficientemente as águas residuárias geradas em sua complexa cadeia produtiva, particularmente em relação à remoção de corantes desses efluentes que, mesmo em pequenas quantidades, conferem cor intensa. Esses efluentes, em sua maioria, apresentam elevadas demandas química e bioquímica de oxigênio, grande quantidade de sólidos suspensos e coloração bastante acentuada, constituindo um dos efluentes mais complexos e problemáticos a serem tratados (AKSU, 2005).

Devido à baixa biodegradabilidade dos corantes, os processos convencionais de tratamento biológico dos efluentes têxteis não são muito eficientes. Usualmente, o tipo de tratamento empregado para remoção do corante envolve processos físicos ou químicos, incluindo coagulação, floculação, oxidação avançada $\mathrm{H}_{2} \mathrm{O}_{2} / \mathrm{UV}$, ozonização, troca iônica, irradiação e adsorção. Algumas dessas técnicas de tratamento têm se mostrado eficientes, embora possuam uma ou outra limitação (FU e VIRARAGHEVAN, 2002; DALLAGO, SMANIOTTO, OLIVEIRA, 2005; AKSU, 2005) seja por questões econômicas ou práticas. A grande vantagem dos processos adsortivos é a possibilidade de recuperação do corante na forma concentrada e a reutilização do adsorvente no processo (KIMURA et al., 1999).

A adsorção é uma das técnicas empregadas com sucesso para remoção efetiva da cor presente em efluentes têxteis. Vários fatores afetam a adsorção, tais como a estrutura molecular ou natureza do adsorvente, a solubilidade do soluto, o pH do meio, e a temperatura e diâmetro molecular do adsorbato (FOUST, 1982). O carvão ativado é o adsorvente mais utilizado na remoção de cor e no tratamento de efluentes têxteis, mas, devido ao seu alto custo, seu uso tem sido reduzido para aplicação em grande escala. Além do mais, o desenvolvimento de adsorventes alternativos de baixo custo tem sido o foco de muitas pesquisas (AKSU, 2001; CALVO et al., 2002; JU et al., 2006; WANG et al., 2008; COSTA et al., 2009; SMITH et al., 2009; RAFATULLAH et al., 2010).

Alguns produtos agrícolas têm sido usados como adsorventes de baixo custo, como semente de girassol (SUN e XINGJING, 1997), polpa de maçã, palha de trigo (ROBINSON et al., 2002), eucalipto (MORAIS et al., 1999), turfa nativa (ALMEIDA et al., 2004) etc., mas eles contêm cargas negativas de celulose, as quais repelem os corantes aniônicos. As fibras celulósicas assumem carga negativa quando submersas em água. Isso tem o efeito de repelir os íons. O eletrólito reduz ou extingue a carga da fibra, facilitando a aproximação do íon corante pelo adsorvente no qual as pontes de hidrogênio ou as forças não polares de Van der Waals podem tornar-se eficazes. Quanto maior for o número de cargas eletronegativas no íon corante, maior a repulsão exercida pela fibra. Esses adsorventes de baixo custo podem ser modificados em
pH ácido para reduzir a repulsão dos íons, mas isso significa um alto custo para o ajuste do $\mathrm{pH}$.

Também foram testados carvão de coque, carvão de pedra, polímero sintético não iônico, terra de diatomácea, bentonita, resinas trocadoras de íons e algodão modificado, vermiculita, pó-de-serra, haste de milho, areia e cascas de arroz. Os resultados demonstraram que, com exceção da vermiculita, mais de 50\% da cor foi removida do efluente. O carvão vegetal e o arroz apresentaram as melhores eficiências (67 e 65\%, respectivamente) (MEYER et al., 1992).

Além desses materiais agrícolas, materiais residuais têm se tornado um objeto de considerável interesse, tais como o lodo resultante da produção do biogás nos biodigestores anaeróbios, quitosana (CHIOU \& LI, 2003; WU et al., 2001), materiais sólidos residuais de diferentes indústrias como cinzas que permanecem no fundo da caldeira, lodo de esgoto sanitário, lamas vermelhas produzidas pelo processamento da bauxita, cinzas resultantes da queima do carvão, lodo residual gerado na etapa de lavagem do gás de alto-forno etc. Esses materiais têm a tendência de remover mais contaminantes inorgânicos do que orgânicos.

Sendo assim, este trabalho teve como objetivo principal, avaliar os parâmetros adsortivos, como temperatura e concentração de eletrólitos na adsorção de corantes reativos mono e bifuncionais, com os sistemas reativos clorotriazina e vinilsulfona, utilizando-se um adsorvente alternativo desenvolvido a partir do lodo do processo de tratamento de efluentes têxteis.

\section{Metodologia}

\section{Preparação das amostras}

O lodo de processo de tratamento de efluentes têxteis foi obtido de uma indústria de beneficiamento têxtil da região de Blumenau (SC).

O lodo adsorvente foi obtido após passar por etapas de ativação térmica e química conforme descrito por Ulson de Souza et al. (2007). A etapa de ativação térmica ocorreu a $500^{\circ} \mathrm{C}$ e, após o aquecimento, a amostra foi ativada quimicamente com ácido acético $1 \mathrm{M}$.

Os três corantes utilizados como adsorbato foram C.I. Reactive Orange 16, C.I. Reactive Red 2 e C.I. Reactive Red 141. Tais corantes foram escolhidos devido à grande quantidade consumida, sendo que os corantes reativos representam cerca de $60 \%$ dos corantes utilizados no mundo, sendo extensivamente empregados no tingimento de fibras têxteis (AL-DEGS et al., 2000).

As estruturas químicas e propriedades desses corantes são apresentadas na Tabela 1. As soluções padrão dos corantes foram preparadas a partir de uma solução-mãe de concentração de $2.000 \mathrm{mg} \cdot \mathrm{L}^{-1}$. Os ensaios espectrofotométricos foram realizados no comprimento de onda de máxima absorção $\left(\boldsymbol{\lambda}_{\text {max }}\right)$, determinado com espectrofotômetro UV-Vis (Cary 50-Varian). Quando necessário, o pH das soluções foi ajustado com ácido acético 0,1 mol.L-1 ou hidróxido de sódio 0,1 mol.L-1. 
Tabela 1 - Propriedades e estruturas químicas dos corantes reativos utilizados

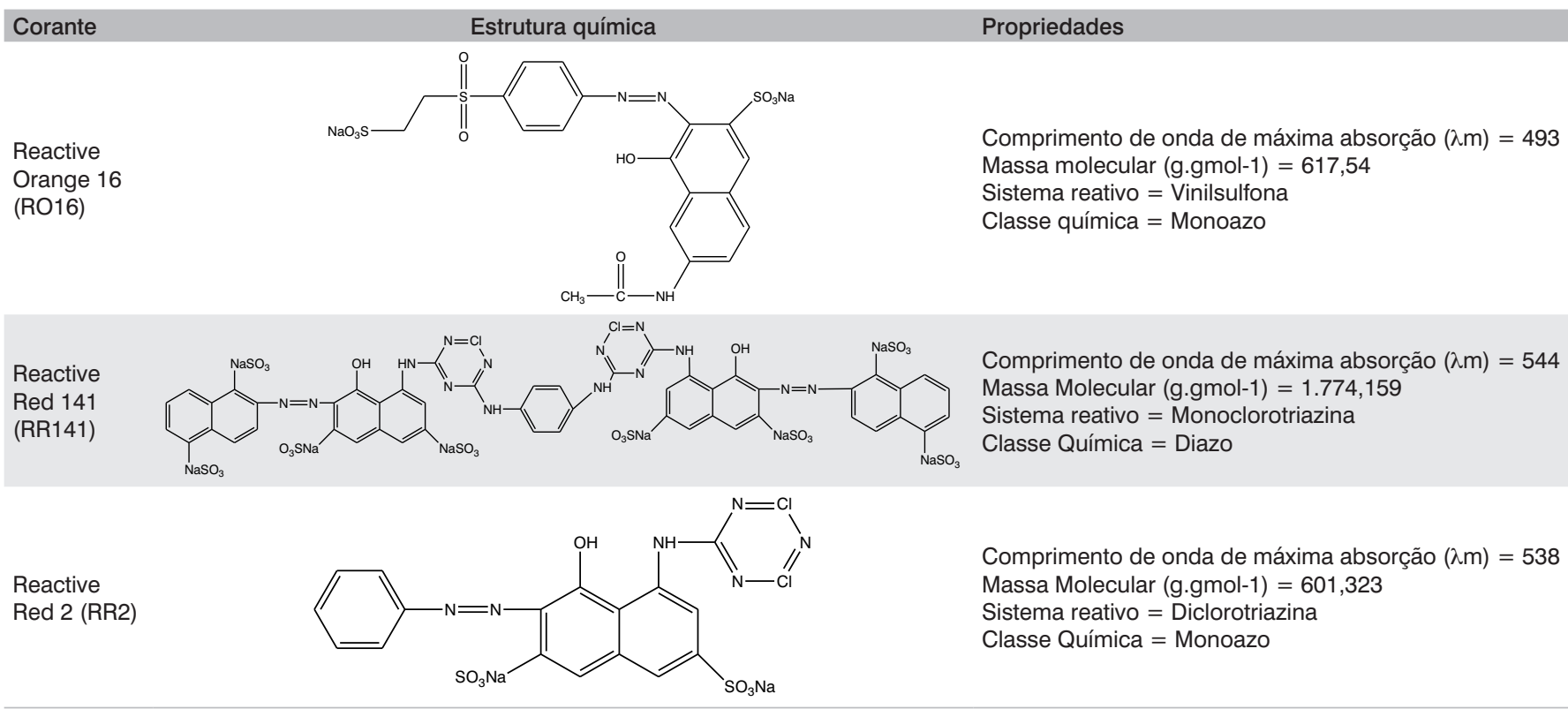

\section{Cinética de adsorção}

A cinética é importante para determinação do tempo em que as amostras atingirão o equilíbrio de adsorção. Para a construção das curvas de cinética de adsorção, adicionou-se 1,0 g de adsorvente a $100 \mathrm{~mL}$ de solução de adsorbato (concentração inicial de $300 \mathrm{mg} . \mathrm{L}^{-1}$ ) em erlenmeyers de $250 \mathrm{~mL}$. O pH dessas amostras foi ajustado para 4,0 com ácido acético 0,1 mol. $\mathrm{L}^{-1}$ e estas foram agitadas em shaker (Modelo TE 420, marca Tecnal). Em intervalos regulares de tempo, foram retiradas alíquotas para análise, até que o equilíbrio fosse atingido e a concentração da solução remanescente fosse determinada por técnicas espectrofotométricas. O procedimento foi realizado para diferentes corantes reativos (azo e diazo), com diferentes sistemas reativos (clorotriazina e vinilsulfona) em distintos parâmetros, como presença ou não de sal à solução e variação da temperatura nos ensaios adsortivos.

\section{Estudos de equilíbrio}

Com o objetivo de conhecer a eficiência do adsorvente, foram determinadas as isotermas de adsorção para diferentes condições experimentais, como temperatura e adição de eletrólitos à solução. As isotermas de adsorção foram determinadas por meio dos ensaios em batelada, nos quais $100 \mathrm{~mL}$ de solução contendo diferentes concentrações de adsorbato (de 100 a $2.000 \mathrm{mg} . \mathrm{L}^{-1}$ ) foram transferidas volumetricamente para erlenmeyers de $250 \mathrm{~mL}$, contendo $1,0 \mathrm{~g}$ do adsorvente, os quais permaneceram sob agitação durante o tempo de equilíbrio obtido pelos ensaios cinéticos, para os três corantes, em diferentes temperaturas e concentrações de sais (cloreto de sódio ou sulfato de sódio).
Os dados obtidos dos experimentos de adsorção foram ajustados de acordo com a isoterma de Langmuir (Equação 1) (ÓRFÃO et al., 2006):

$q_{e q}=\left(q_{\max } K_{L} C_{e q}\right) /\left(1+K_{L} C_{e q}\right)$

Equação 1

sendo:

$q_{\text {eq }}$ : quantidade de corante adsorvida no equilíbrio por unidade de massa de adsorvente (mg. $\mathrm{g}^{-1}$ );

$q_{\max }:$ quantidade máxima de corante adsorvida por unidade de massa de adsorvente (mg. $\left.\mathrm{g}^{-1}\right)$;

$K_{L}$ : constante de equilíbrio de Langmuir, $\left(\mathrm{L}^{-g^{-1}}\right)$;

$\mathrm{C}_{\text {eq }}$ : concentração de corante na solução no equilíbrio (mg.L-1.1).

O modelo de isoterma de equilíbrio é teórico e baseado na hipótese de que as forças de interação entre as moléculas adsorvidas são desprezíveis e que cada sítio pode ser ocupado por apenas uma molécula. Todas as moléculas são adsorvidas sobre um número fixo e definido de sítios ativos (KUMAR e SIVANESAN, 2006).

As características essenciais de uma isoterma de Langmuir podem ser expressas em termos de um fator de separação adimensional constante ou comumente chamado de parâmetro de equilíbrio $\left(\mathrm{R}_{\mathrm{L}}\right)$, que é definido pela Equação 2. Quando o valor de $\mathrm{R}_{\mathrm{L}}$ está entre 0 e $1\left(0<R_{\mathrm{L}}<1\right)$, isso indica que tipo de isoterma de adsorção é favorável (CIOLA, 1981).

$R_{L}=1 /\left(1+K_{L} \cdot C_{e}\right)$

Equação 2

sendo:

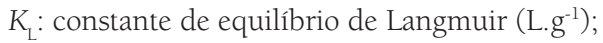

$C_{e}$ : concentração de corante no equilíbrio $\left(\mathrm{mg} \cdot \mathrm{L}^{-1}\right)$. 


\section{Resultados e Discussão}

\section{Cinética de adsorção}

A Figura 1 apresenta as curvas de cinética de adsorção comparativas para o corante RO16, RR2 e RR141 conduzidas em diferentes temperaturas.

Comparando os três corantes a $25^{\circ} \mathrm{C}$ (Figura 1A), percebe-se maior eficiência do adsorvente para a remoção do corante RR141. A concentração de corante remanescente no equilíbrio para o corante RO16 foi de $10,71 \mathrm{mg} \cdot \mathrm{L}^{-1}$, enquanto que para o corante RR2 a essa temperatura foi de 18,69mg. $\mathrm{L}^{-1}$, e para o corante RR141 foi de somente 3,76mg. $\mathrm{L}^{-1}$. Esses resultados indicam que os corantes com maior quantidade de cargas têm maior tendência em serem adsorvidos pelo adsorvente devido à atração elétrica dos corantes pelos sítios de cargas positivas formadas pela dupla camada elétrica do adsorvente.

A dupla camada elétrica forma-se quando íons metálicos presentes no lodo são calcinados. Óxidos metálicos são formados, os quais, em solução são hidratados formando íons hidróxidos, que são responsáveis pela captura de íons positivos pela superfície do adsorvente. Essa camada é formada quando duas fases químicas diferentes são colocadas em contato, com uma diferença aparente no potencial elétrico na interface. Essa diferença de potencial leva a uma separação de cargas na qual a superfície carregada positivamente ou negativamente atrai íons de cargas opostas e repele íons de mesma carga. A separação das cargas causa a distribuição dos íons na superfície e origina a dupla camada elétrica, formada pela superfície carregada eletricamente, pelos íons de cargas opostas e pelos íons de mesma carga, os quais são difusos no meio (camada difusa). Portanto, o adsorvente é negativo e, em contato com a solução, cria-se uma camada positiva ao redor do adsorvente.

A adição de sais pode aumentar ou diminuir a capacidade de adsorção dependendo da dimerização dos corantes em solução. Um número de forças intermoleculares tem sido sugerido para explicar essa agregação; essas forças incluem: força de Van der Waals, forças ín-dipolo e forças dipolo-dipolo as quais ocorrem entre as moléculas de corante em solução. Um aumento, por exemplo, na capacidade de adsorção se dá devido à agregação de moléculas induzidas pela adição de íons sal, isto é, a força dos íons sal para agregar as moléculas de corante aumenta a extensão da sorção pela superfície do adsorvente.

As estruturas do RO16, RR2 e do RR141 possuem 3, 2 e 8 grupos sulfônicos $\left(-\mathrm{SO}_{3}{ }^{-}\right)$respectivamente. Então, o RR141, com uma maior quantidade de cargas negativas, têm maior força iônica para ser atraído pelas cargas positivas da dupla camada elétrica do adsorvente. Devido a esse mesmo fato, foi possível verificar a semelhança de resultados para os corantes RO16 e RR2, que possuem quantidades praticamente equivalentes de grupos sulfona, porém são organizados de forma diferenciada em suas estruturas, sendo corantes de diferentes sistemas reativos. O tempo de equilíbrio para os ensaios realizados a 25 e $40^{\circ} \mathrm{C}$ (Figuras $1 \mathrm{~A}$ e B) não ultrapassou 5 horas (300 minutos) para os corantes RR2 e RR141. O tempo da cinética de adsorção a $60^{\circ} \mathrm{C}$ (Figura 1C) foi mais prolongado para o corante RO16, sendo de aproximadamente 6 horas (360 minutos), enquanto que, para os outros dois corantes, não ultrapassou 5,5 horas (330 minutos).

Na Figura 2 são apresentadas as curvas de cinética de adsorção comparativas para o corante RO16 (A), RR2 (B) e para o corante RR141 (C), na presença ou ausência de cloreto de sódio ou sulfato de sódio à solução.

Para os ensaios de cinética de adsorção apresentados na Figura $3 \mathrm{~A}$, o tempo de equilíbrio para o corante RO16 foi o de maior duração, levando em média 6 horas (360 minutos) quando adicionadas diferentes concentrações de sais à solução, enquanto que em (B) e (C) não ultrapassou 5,5 horas (330 minutos) sendo de 5 horas (300 minutos) para o corante RR141 na presença de 10\% (\% massa) de cloreto de sódio e de 4 horas (240 minutos) para o corante RR2 na ausência de sal.

$\mathrm{Na}$ Figuras 3A e B, é possível observar que quando adicionado cloreto de sódio $(\mathrm{NaCl})$ ao adsorbato RO16 ou RR2 em diferentes concentrações, 1, 5 e 10\% (m:m), verifica-se uma redução da capacidade adsortiva do adsorvente em comparação à adição de sulfato de sódio para ambos os corantes. Há uma maior redução da capacidade adsortiva à medida que se aumenta a concentração de $\mathrm{NaCl}$ para o corante RR2. O corante RO16 não demonstra redução significativa na capacidade de adsorção em relação à variação da concentração de $\mathrm{NaCl}$, demonstrando mesmo comportamento adsortivo seja qual for a concentração desse sal.

Quando se adicionam 10\% de $\mathrm{NaCl}$ à solução em (B), a capacidade adsortiva é reduzida em aproximadamente 77,64\% e para (A) essa redução foi de 78,43\% com relação à concentração de equilíbrio obtida quando não se adiciona nenhuma espécie de sal à solução. Esse comportamento poderia ser justificado pelo fato do grande aumento no número de eletrólitos de carga positiva, sendo repelidos quando em contato com as cargas positivas do adsorvente impedindo assim, que os íons negativos do corante se aproximem da superfície do mesmo. A adição de sulfato de sódio confere à solução uma alta concentração de íons sulfato, que neutralizam os cátions da superfície do adsorvente. Isso faz com que os ânions do corante se aproximem mais intensamente da superfície do adsorvente, possibilitando um menor efeito inibidor da adsorção comparativamente ao íon cloreto.

Tal efeito é ainda melhor observado para o corante RO16, que teve um aumento significativo na capacidade de adsorção do adsorvente na presença do sulfato de sódio, na qual o potencial aumentou gradativamente conforme o aumento na concentração do eletrólito. Observa-se a grande influência dos ânions no processo de redução da capacidade de adsorção, no caso em estudo o $\mathrm{Cl}^{-}$e o $\mathrm{SO}_{4}^{-2}$, sendo muito maior esse efeito no caso do primeiro, comportamento este também investigado por Netpradit et al. (2004). 


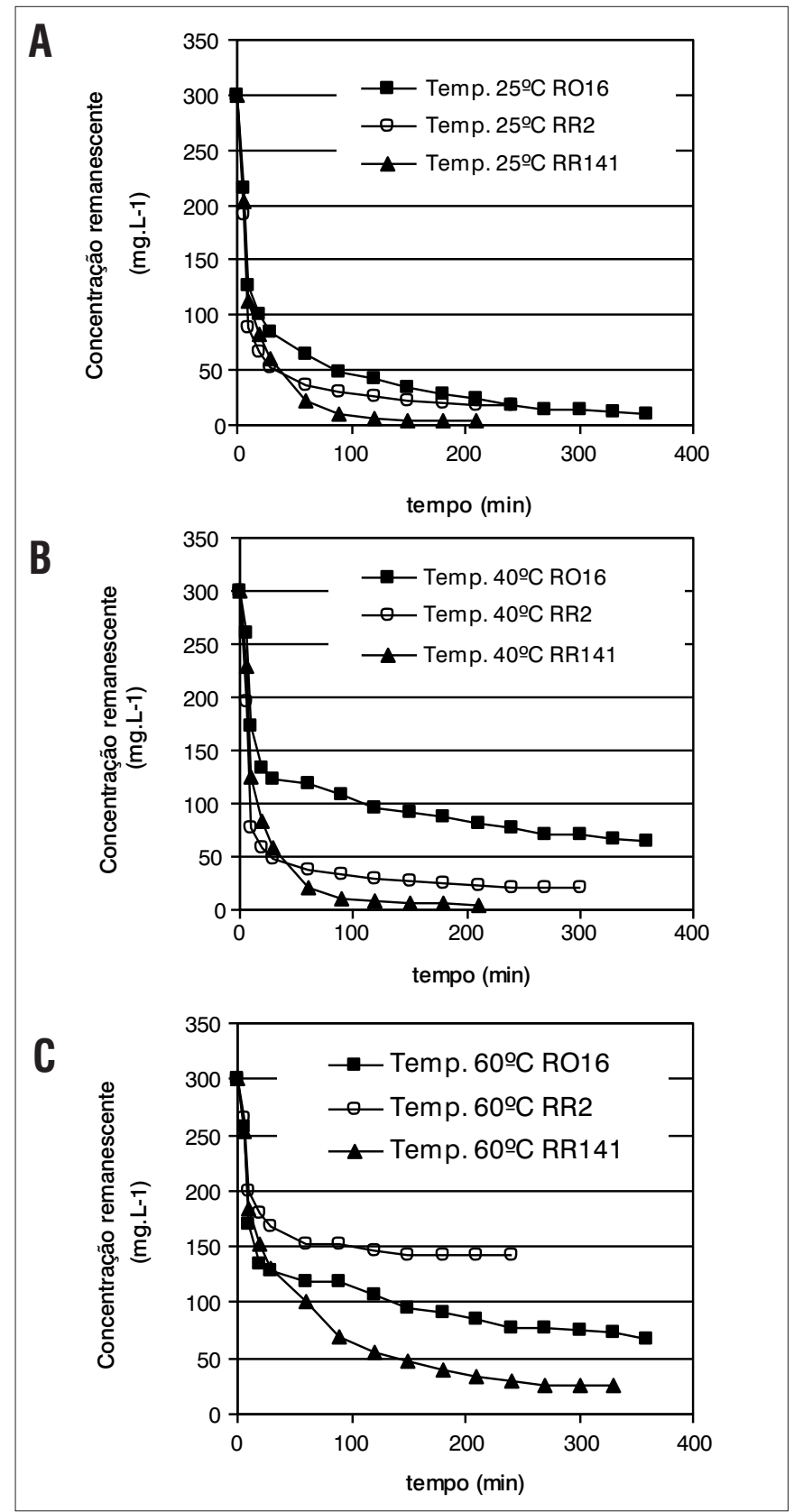

Figura 1 - Curvas de cinética de adsorção comparativas para o corante R016, RR2 e RR141 conduzidas em diferentes temperaturas (a) $25^{\circ} \mathrm{C}$, (b) $40^{\circ} \mathrm{C}$ e (c) $60^{\circ} \mathrm{C}$.

A influência do carregamento da superfície do adsorvente afeta marcantemente o desempenho do processo de adsorção, sendo a medida do potencial Zeta reveladora quanto à natureza da carga. A medida do potencial Zeta é função do valor do $\mathrm{pH}$, permitindo assim estimar se a adsorção de cátions ou ânions é favorecida. Quando o pH empregado no processo de adsorção for igual ao pHzpc (ponto de carga 0) a superfície do material adsorvente é neutra. Valores de pH superiores ao pHzpc sugerem que a superfície está carregada negativamente e a adsorção de cátions é favorecida. Para valores de $\mathrm{pH}$ inferiores ao $\mathrm{pHzpc}$ a superfície se apresenta carregada positivamente e, nesse caso, a adsorção de
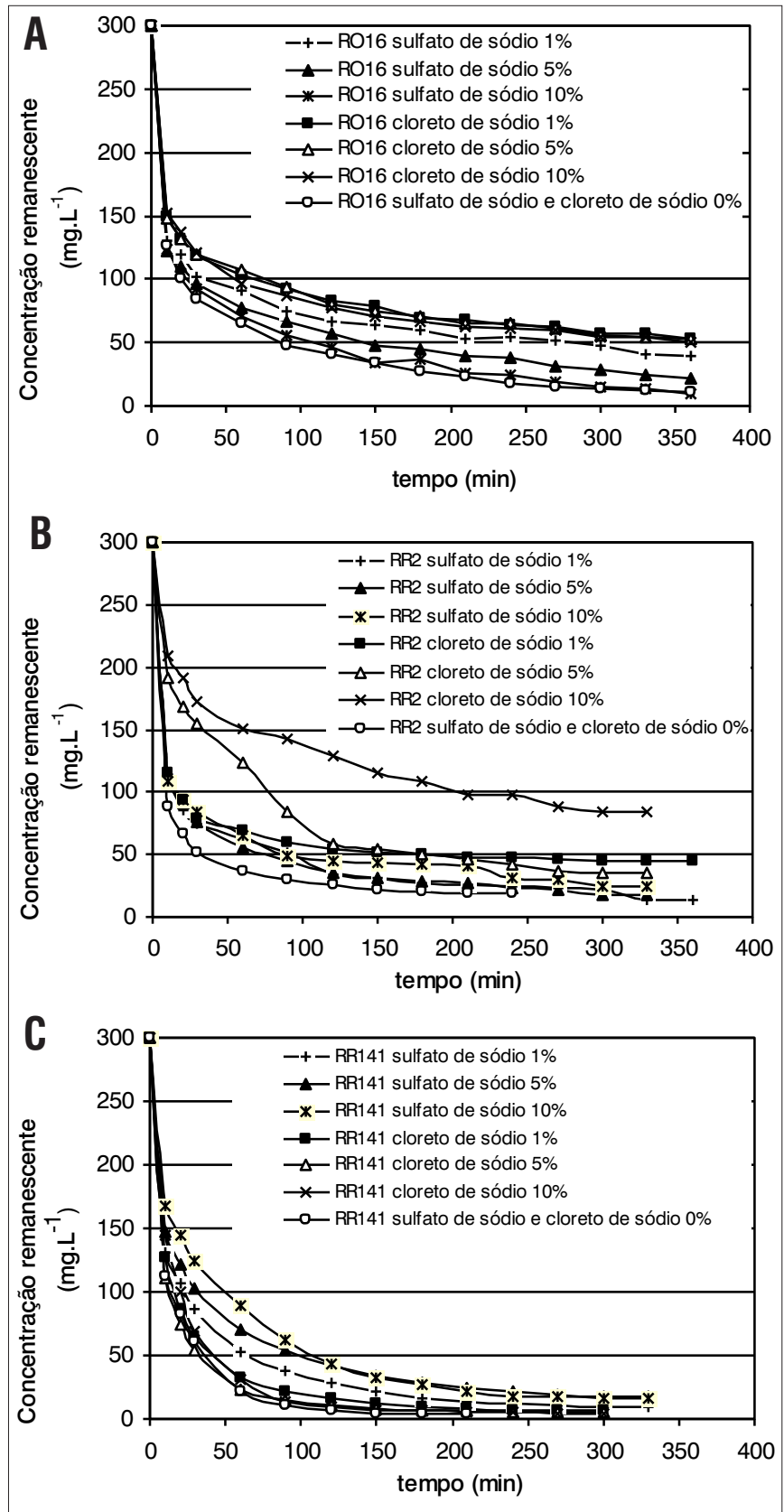

Figura 2 - Curvas de cinética de adsorção comparativas para o corante R016 (A), RR2 (B) e para o corante RR141 (C), na presença ou ausência de cloreto de sódio ou sulfato de sódio à solução.

ânions é favorecida como discutido por Al-Degs et al. (2000) e Al-Degs et al. (2008)

É possível estabelecer uma relação com o estudo reportado por Al-Degs et al. (2000) no qual o carvão ativado F-400 foi usado para remoção de corantes reativos. $\mathrm{O}$ valor do $\mathrm{pH}_{z \mathrm{pc}}$ para esse carvão foi 7,2 e os valores do $\mathrm{pH}$ medidos no equilíbrio para os três corantes reativos estudados foram 5,5, 5,0 e 5,0 para o amarelo, preto e vermelho, respectivamente. Baixos valores de $\mathrm{pH}$ indicam fortemente que o carvão ativado F-400 tem carga positiva na camada externa (no equilíbrio $\mathrm{pH}<\mathrm{pH}_{\mathrm{zpc}}$ ) durante o processo de adsorção. Essas cargas positivas atacam as porções negativas dos corantes reativos. Esse 
comportamento também foi observado por Netpradit et al. (2004), o qual obteve um $\mathrm{pH}_{\text {zpc }}$ por volta de 8,7 , favorecendo a captura de cargas negativas dos corantes reativos.

Kimura et al. (1999), em um estudo sobre o efeito do $\mathrm{pH}$ e do tempo de contato na adsorção de corantes reativos por microesferas de quitosana, analisaram gráficos que ilustraram isotermas de adsorção em função dos parâmetros pH, tempo de contato e capacidade de adsorção e concluíram que a adsorção em meio ácido dos corantes Preto 5 e Laranja 16 é atribuída, principalmente, às interações iônicas entre os grupos $-\mathrm{SO}^{-3}$ dos corantes e $-\mathrm{NH}^{3+}$ da quitosana. Portanto, a acidez do meio é um fator importante, influenciando as interações entre adsorvente e adsorbato no que diz respeito a interações eletrostáticas.

Para o corante RR141, a adição de íons sulfato à solução desfavoreceu acentuadamente a adsorção, pois esse corante possui um maior número ânions interagindo com os íons positivos do sódio e impedindo uma aproximação do corante aniônico até a superfície de dupla camada do adsorvente. Esse comportamento pode ser visualizado na Figura 2C, a qual apresenta as cinéticas comparativas para o corante RR141 na presença ou ausência de cloreto de sódio ou sulfato de sódio à solução. A adição do cloreto de sódio (10\%) favoreceu a adsorção no caso do corante RR141, sendo este mais aniônico que o RO16 e o RR2, os cátions do sal podem se ligar com o adsorbato negativo, reduzindo a repulsão iônica, aumentando, assim, a quantidade de moléculas adsorvidas na superfície.

\section{Estudo de equilíbrio}

A isoterma de adsorção mostra a relação de equilíbrio do adsorbato no adsorvente e na solução. As isotermas de adsorção indicam como o adsorvente efetivamente adsorverá as impurezas presentes e se a purificação requerida pode ser obtida, bem como apresenta uma estimativa máxima da capacidade de adsorção (FERNANDES, 2005).

Os valores dos parâmetros de ajuste dos modelos de Langmuir são apresentados na Tabela 2

Ao avaliar os parâmetros obtidos (Tabela 2), por meio da linearização da equação de Langmuir (Equação 1), verifica-se que a capacidade máxima de adsorção do lodo adsorvente $\left[q_{\max }\left(m g \cdot g^{-1}\right)\right]$ diminui à medida que se aumenta a temperatura dos ensaios adsortivos para os três corantes, aumentando em pequena quantidade apenas para o corante RO16, na temperatura de 40 para $60^{\circ} \mathrm{C}$. Quando se trabalha com diferentes concentrações de cloreto de sódio adicionados ao adsorbato RO16 e RR2, há diminuição na capacidade máxima de adsorção segundo Langmuir $\left(\mathrm{q}_{\max }\right)$, sendo mais expressiva gradativamente para o adsorbato RR2. Esse comportamento foi observado nos ensaios cinéticos (Figura 3A). Para o corante RR141 há um aumento da capacidade máxima de adsorção à medida que se aumenta a concentração de $\mathrm{NaCl}$, comportamento este também verificado nos ensaios cinéticos (Figura 3B). Para os ensaios realizados com adição de cloreto de sódio para os três corantes estudados, foram obtidos coeficientes de correlação que variaram entre 0,907 e 0,992 e, para os ensaios realizados com adição de sulfato de sódio para os três corantes estudados, os coeficientes de correlação variaram entre 0,862 e 0,997

Quando adicionadas diferentes concentrações em massa de sulfato de sódio ao adsorbato, a eficiência do processo adsortivo $\left(q_{\max }\right.$ de Langmuir) para o corante RO16 e RR2 aumentou, em relação à adição de $\mathrm{NaCl}$. Para o corante RR141 há uma pequena redução da quantidade máxima de corante adsorvida por grama de adsorvente, quando comparado aos ensaios adsortivos onde foi adicionado $\mathrm{NaCl}$ à solução. Esse comportamento foi identificado nos ensaios cinéticos conforme apresentado na Figura 3C.

A partir da Tabela 2 foram determinadas as melhores condições adsortivas pelo maior valor de $\mathrm{q}_{\max }\left(\mathrm{mg} \cdot \mathrm{g}^{-1}\right)$ obtido por ajuste de Langmuir. Para o corante RO16 a melhor condição de adsorção é a $25^{\circ} \mathrm{C}$ na ausência completa de sal ao adsorbato. Tal conclusão se deve ao fato desse corante se comportar semelhantemente ao corante RR2 e também pelo fato desse resultado já ser comprovado nas cinéticas apresentadas na Figura 2C.

Tabela 2 - Parâmetros de equilíbrio da isoterma de Langmuir para diferentes condições experimentais

\begin{tabular}{|c|c|c|c|c|}
\hline Corantes & Temperatura $\left({ }^{\circ} \mathrm{C}\right)$ & $\frac{\mathrm{K}_{\mathrm{L}}}{\left(\mathrm{L} \cdot \mathrm{mg}^{-1}\right)^{*}}$ & $\underset{\left(m g \cdot g^{-1}\right)^{\star *}}{\mathrm{q}_{\max }}$ & $\mathrm{R}^{2}$ \\
\hline R016 & $\begin{array}{l}25 \\
40 \\
60\end{array}$ & $\begin{array}{l}0,012 \\
0,016 \\
0,008\end{array}$ & $\begin{array}{l}81,30 \\
52,63 \\
57,14\end{array}$ & $\begin{array}{l}0,930 \\
0,996 \\
0,959\end{array}$ \\
\hline RR2 & $\begin{array}{l}25 \\
40 \\
60\end{array}$ & $\begin{array}{l}0,116 \\
0,275 \\
0,077\end{array}$ & $\begin{array}{l}53,476 \\
31,746 \\
24,876\end{array}$ & $\begin{array}{l}0,928 \\
0,872 \\
0,954\end{array}$ \\
\hline RR141 & $\begin{array}{l}25 \\
40 \\
60\end{array}$ & $\begin{array}{l}0,209 \\
0,199 \\
0,117\end{array}$ & $\begin{array}{l}66,670 \\
65,789 \\
51,020\end{array}$ & $\begin{array}{l}0,997 \\
0,976 \\
0,984\end{array}$ \\
\hline Corantes & $\begin{array}{l}\text { Concentração de } \mathrm{NaCl} \\
\text { (m:m) (\%) T=25C }\end{array}$ & $\begin{array}{c}\mathrm{K}_{\mathrm{L}} \\
\left(\mathrm{L} \cdot \mathrm{mg}^{-1}\right)^{*}\end{array}$ & $\underset{\left(m g \cdot g^{-1}\right)^{\star \star}}{\mathrm{q}_{\max }}$ & $\mathrm{R}^{2}$ \\
\hline RO16 & $\begin{array}{c}1 \\
5 \\
10\end{array}$ & $\begin{array}{l}0,006 \\
0,008 \\
0,010\end{array}$ & $\begin{array}{l}72,99 \\
60,60 \\
60,60\end{array}$ & $\begin{array}{l}0,992 \\
0,981 \\
0,956\end{array}$ \\
\hline RR2 & $\begin{array}{c}1 \\
5 \\
10\end{array}$ & $\begin{array}{l}0,104 \\
0,366 \\
0,409\end{array}$ & $\begin{array}{l}45,450 \\
30,120 \\
22,026\end{array}$ & $\begin{array}{l}0,907 \\
0,930 \\
0,931\end{array}$ \\
\hline RR141 & $\begin{array}{c}1 \\
5 \\
10\end{array}$ & $\begin{array}{l}0,210 \\
0,091 \\
0,077\end{array}$ & $\begin{array}{l}44,248 \\
47,619 \\
78,740\end{array}$ & $\begin{array}{l}0,987 \\
0,967 \\
0,984\end{array}$ \\
\hline Corantes & $\begin{array}{c}\text { Concentração de sulfato } \\
\text { de sódio } \\
\text { (m:m) (\%) } \mathrm{T}=25 \% \mathrm{C}\end{array}$ & $\begin{array}{c}\mathrm{K}_{\mathrm{L}} \\
\left(\mathrm{L} \cdot \mathrm{mg}^{-1}\right)^{*}\end{array}$ & $\underset{\left(m g \cdot g^{-1}\right)^{\star \star}}{q_{\max }}$ & $\mathrm{R}^{2}$ \\
\hline RO16 & $\begin{array}{c}1 \\
5 \\
10\end{array}$ & $\begin{array}{l}0,005 \\
0,039 \\
0,015\end{array}$ & $\begin{array}{l}81,30 \\
55,55 \\
62,11\end{array}$ & $\begin{array}{l}0,997 \\
0,921 \\
0,958\end{array}$ \\
\hline RR2 & $\begin{array}{c}1 \\
5 \\
10\end{array}$ & $\begin{array}{l}0,051 \\
0,291 \\
0,191\end{array}$ & $\begin{array}{l}47,393 \\
39,841 \\
39,526\end{array}$ & $\begin{array}{l}0,985 \\
0,965 \\
0,991\end{array}$ \\
\hline RR141 & $\begin{array}{c}1 \\
5 \\
10\end{array}$ & $\begin{array}{l}0,347 \\
0,750 \\
0,004\end{array}$ & $\begin{array}{l}52,356 \\
40,161 \\
66,667\end{array}$ & $\begin{array}{l}0,912 \\
0,862 \\
0,960\end{array}$ \\
\hline
\end{tabular}

${ }^{*} \mathrm{~K}_{\mathrm{L}}$ : constante de equilíbrio de Langmuir $\left(\mathrm{L} . \mathrm{mg}^{-1}\right)$; ${ }^{* *} \mathrm{q}_{\max }$ : quantidade máxima de corante adsorvida por unidade de massa de adsorvente $\left(\mathrm{mg}^{-1} \mathrm{~g}^{-1}\right)$; 
Tabela 3 - Percentuais de remoção para o corante R016, RR2 e RR141 para as melhores condições adsortivas, nas concentrações iniciais de 100 e $500 \mathrm{mg} \cdot \mathrm{L}^{-1}$

\begin{tabular}{|c|c|c|c|c|c|c|}
\hline Adsorbato & $\begin{array}{l}\text { Temp. } \\
\left({ }^{\circ} \mathrm{C}\right)\end{array}$ & $\frac{\mathrm{C}_{\mathrm{o}}}{\left(\mathrm{mg} \cdot \mathrm{L}^{-1}\right)^{*}}$ & $\frac{\mathrm{C}_{\mathrm{e}}}{\left(\mathrm{mg} \cdot \mathrm{L}^{-1}\right)^{\star *}}$ & $\underset{\left(m g \cdot g^{-1}\right)^{\star \star \star \star}}{\mathrm{q}}$ & \% Remoção & $\mathrm{R}_{\mathrm{L}}{ }^{* \star \star \star}$ \\
\hline \multirow[t]{2}{*}{ RO16 } & \multirow[t]{2}{*}{25} & 500 & 118,549 & 38,15 & 76,29 & 0,4169 \\
\hline & & 100 & 1,383 & 9,86 & 98,61 & 0,9839 \\
\hline \multirow[t]{2}{*}{ RR2 } & \multirow[t]{2}{*}{25} & 500 & 57,14 & 44,29 & 88,57 & 0,1313 \\
\hline & & 100 & 0,274 & 9,97 & 99,73 & 0,9693 \\
\hline \multirow[t]{2}{*}{ RR141 com $10 \%$ em massa de cloreto de sódio } & \multirow[t]{2}{*}{25} & 500 & 21,37 & 47,86 & 95,72 & 0,3783 \\
\hline & & 100 & 2,80 & 9,72 & 97,20 & 0,8230 \\
\hline
\end{tabular}

${ }^{*} \mathrm{C}_{\mathrm{o}}$ : concentração inicial de corante $\left(\mathrm{mg} \cdot \mathrm{L}^{-1}\right) ;{ }^{* *} \mathrm{C}_{\mathrm{e}}$ : concentração de corante na solução em equilíbrio ou no breakthrough (mg.L-1); *** $\mathrm{q}_{\mathrm{e}}$ : quantidade de corante adsorvida no equilíbrio por unidade de massa de adsorvente (mg.g-1); ${ }^{\star \star \star \star} R_{L}$ : parâmetro de equilíbrio de Langmuir (adimensional).

Os valores de $\mathrm{q}_{\max }\left(\mathrm{mg} \cdot \mathrm{g}^{-1}\right)$ para as melhores condições experimentais para os três corantes estudados foram $81,30 \mathrm{mg} \cdot \mathrm{g}^{-1}$ para o corante RO16 a $25^{\circ} \mathrm{C}$ na ausência de sais, $53,476 \mathrm{mg}$. $\mathrm{g}^{-1}$ para o corante RR2 a $25^{\circ} \mathrm{C}$ na ausência de sais e de $78,740 \mathrm{mg} \cdot \mathrm{g}^{-1}$ para o corante RR141 a $25^{\circ} \mathrm{C}$ na presença de $10 \%$ de cloreto de sódio ao adsorbato.

Kimura et al. (1999), num estudo sobre efeito do pH e do tempo de contato na adsorção de corantes reativos por microesferas de quitosana, analisaram gráficos que ilustram isotermas de adsorção em função dos parâmetros $\mathrm{pH}$, tempo de contato e capacidade de adsorção, e concluíram que a adsorção em meio ácido dos corantes Preto 5 e Laranja 16 é atribuída, principalmente, às interações iônicas entre os grupos $-\mathrm{SO}^{-3}$ dos corantes e $-\mathrm{NH}^{3+}$ da quitosana. Portanto, a acidez do meio é um fator importante, influenciando as interações entre adsorvente e adsorbato no que diz respeito a interações eletrostáticas (KIMURA et al., 1999).

Os valores de capacidade máxima de adsorção obtidos com o uso do adsorvente proposto por este trabalho podem ser comparados com os valores apresentados por Netpradit et al. (2003), os quais estudaram a remoção de corantes reativos azo, CI RR2, CI RR120 e CI RR141 por lodo de hidróxido metálico seco e obtiveram valores de $\mathrm{q}_{\max }$ muito próximos dos resultados aqui encontrados. Os experimentos foram conduzidos à temperatura de $30^{\circ} \mathrm{C}$ e os valores de $\mathrm{q}_{\max }$ encontrados por ajuste de Langmuir para os corantes RR2 e RR141 foram 62,50mg.g $\mathrm{g}^{-1}$ e $56,18 \mathrm{mg} \cdot \mathrm{g}^{-1}$, respectivamente.

As características essenciais de uma isoterma de Langmuir podem ser expressas em termos de um fator de separação adimensional constante ou comumente chamado de parâmetro de equilíbrio, $\mathrm{R}_{\mathrm{L}}$ obtido pela Equação 2. Para as melhores condições adsortivas, para os três corantes estudados, $\mathrm{R}_{\mathrm{L}}$ variou de 0,13 a 0,98 , indicando que essas isotermas são favoráveis, conforme Ciola (1981).

$\mathrm{Na}$ Tabela 3 são apresentados os percentuais de remoção para o corante RO16, RR2 e RR141 para as melhores condições experimentais de adsorção nas concentrações iniciais de corantes de 100 e 500mg. $\mathrm{L}^{-1}$.

Pelos dados de porcentual de remoção apresentados na Tabela 3, pode-se verificar a grande efetividade de remoção de cor pelo adsorvente. Para concentrações maiores que $100 \mathrm{mg} . \mathrm{L}^{-1}$, a porcentagem de remoção se reduz para os três corantes estudados.

\section{Conclusões}

Quando os ensaios são conduzidos à temperatura de $25^{\circ} \mathrm{C}$, o corante RR141 é mais facilmente adsorvido pelo adsorvente em relação ao adsorbato RO16 e RR2. Isso se deve a diferentes estruturas químicas que os três corantes apresentam.

Quanto aos ensaios cinéticos, verifica-se que quando se adiciona cloreto de sódio ao adsorbato RO16, seja qual for a concentração, a capacidade adsortiva é reduzida. Para o RR2 essa redução acontece apenas para concentrações acima de 5\% de cloreto de sódio e gradativamente. Para o corante RR141, a adição de $\mathrm{NaCl}$ aumentou a capacidade de adsorção no equilíbrio de 44,25mg.g . $^{-1}$ ( $\%$ em massa de $\mathrm{NaCl}$ ) para $78,74 \mathrm{mg} \cdot \mathrm{g}^{-1}$ (10\% em massa de $\mathrm{NaCl}$ ).

Para o corante RO16, a presença de sulfato de sódio favoreceu a adsorção, ao contrário do que acontece com os outros dois corantes (RR2 e RR141), que não obtiveram melhoras com a adição deste. Isso se explica pelo fato dos corantes RR2 e RR141 terem uma menor quantidade de íons condutores na presença de 1, 5 e 10\% de sulfato de sódio à solução.

Mediante realização dos ensaios de equilíbrio, foi possível concluir que o maior valor de capacidade adsortiva obtido para o corante RO16 e RR2 foi à temperatura de $25^{\circ} \mathrm{C}$ na ausência de sais adicionados à solução e para o corante RR141, foi a $25^{\circ} \mathrm{C}$ na presença de $10 \%$ em massa de cloreto de sódio à solução. Os resultados de $\mathrm{q}_{\max }\left(\mathrm{mg} \cdot \mathrm{g}^{-1}\right)$ obtidos por ajuste de Langmuir foram de $81,30 \mathrm{mg} \cdot \mathrm{g}^{-1}$; $53,48 \mathrm{mg} \cdot \mathrm{g}^{-1}$ e $78,74 \mathrm{mg} \cdot \mathrm{g}^{-1}$, respectivamente, para as melhores condições experimentais.

Verificou-se a grande efetividade de remoção de cor do adsorvente para os três corantes estudados, na qual a porcentagem de remoção para o corante RO16, RR2 e RR141 para as melhores condições adsortivas, nas concentrações iniciais de 100 e 500mg. $\mathrm{L}^{-1}$ foi de $76,29 \%$ e $98,61 \%$, respectivamente, para o corante RO16, enquanto que para o corante RR2 foi de $99,73 \%$ e $88,57 \%$, respectivamente, e de $97,20 \%$ e 95,72\%, respectivamente, para o corante RR141.

\section{Agradecimentos}

À Universidade Regional de Blumenau (FURB) pelo suporte físico na realização do trabalho e pelo apoio financeiro. 


\section{Referências}

AKSU, Z. Application of biosorption for the removal of organic pollutants: a review. Process Biochemistry, v. 40, p. 997-1026, 2005.

AKSU, Z. Biosorption of reactive dyes by dried activated sludge: equilibrium and kinetic modeling. Biochemical Engineering Journal, v. 7, p. 79-84, 2001.

AL-DEGS, Y.S. et al. Effect of solution $\mathrm{pH}$, ionic strength, and temperature on adsorption behavior of reactive dyes on activated carbon. Dyes and Pigments, v. 77, p. 16-23, 2008

AL-DEGS, Y.S. et al. Effect of carbon surface chesmistry on the removal of reactive dyes from textile effluent. Water Research, v. 34, n. 3, p. 927-935, 2000.

ALMEIDA, A.C.M.; PEDRO, R.J.; CORSO, C.R. Remoção de corante têxtil por turfa nativa através de adsorção em diferentes concentrações hidrogeniônicas. Arquivos do Instituto Biológico, v. 71, p. 1-749, 2004

BUZZINI, A. P. et al. Evaluation of aerobic and anaerobic treatment of Kraft pulp mill effluent for organochlorines removal. Water Practice and Technology, v. 1, p. 1-8, 2006.

Calvo, L.F. et al. Upgrading sewage sludges for adsorbent preparation by different treatments, Bioresource Technology, v. 80, p. 143-148, 2002

CHIOU, M.S.; LI, H.Y., Adsorption behavior of reactive dye in aqueous solution on chemical cross-linked chitosan beads. Chemosphere, v. 50, p. 1095-1105, 2003

CIOLA, R. Fundamentos de catálise. São Paulo: Edusp, 1981.

COSTA, E.P. et al. Uso da casca de arroz como adsorvente na remoção do corante têxtil Vermelho Remazol 5R. Cadernos de Pesquisa, v. 16, n. 2, p. 44-50, 2009.

DALLAGO, R.M.; SMANIOTTO, A.; OLIVEIRA, L.C.A. Resíduos sólidos de curtumes como adsorventes para a remoção de corantes em meio aquoso. Química Nova, v. 28, n. 3, p. 433-437, 2005.

FERNANDES, R. Adsorventes alternativos para remoção de fenol em solução aquosa. Dissertação [Mestrado]. Santa Catarina: Universidade Federal de Santa Catarina, 2005.

FOUST, A.S.; WENZEL, L.A.; CLUMP, C.W. Princípio das operações unitárias. Rio de Janeiro: Guanabara Dois, 1982.

FU, Y.; VIRARAGHEVAN, T. Removal of Congo Red from an aqueous solution by fungus Aspergillus niger. Advances in Environmental Research, v. 7, p. 239-247, 2002.

JU, D.J. et al. Biosorption characteristics of reactive dye onto dried activated sludge. Water Practice \& Technology, v. 1, n. 3, 2006.

KIMURA, Y.I. et al. Efeito do pH e do tempo de contato na adsorção de corantes reativos por microesferas de quitosana. Polímeros: Ciência e Tecnologia, p. 51-57, 1999.
KUMAR, K.V.; SIVANESAN, S. Equilibrium data, isotherm parameters and process design for partial and complete isotherm of methylene blue onto activated carbon. Journal of Hazardous Materials, v. B134, p. 237 244, 2006

MEYER, V.; CARLSSON, F.H.H.; OELLERMANN, R.A. Decolourization of textile effluent using a low cost natural adsorvent material. Water Science Technology, v. 26, n. 5-6, p. 1205-1211, 1992.

MORAIS, L. C. et al. Reactive dyes removal from wastewaters by adsorption on eucalyptus bark: variables that define the process. Water Research, v. 33, p. 979-988, 1999.

NETPRADIT, S.; THIRAVETYAN, P.; TOWPRAYOON, S. Application of waste metal hydroxide sludge for adsorption of azo reactive dyes. Water Research, v. 37, p. 763-772, 2003.

Adsorption of three azo reactive dyes by metal hydroxide sludge: effect of temperature, $\mathrm{pH}$ and electrolytes. Colloid and Interface Science, v. 270, p. $255-261,2004$.

ÓRFÃO, J. J. M. et al. Adsorption of a reactive dye on chemically modified activated carbons-Influence of Ph. Journal of Colloid and Interface Science, v. 296, p. 480-489, 2006

RAFATULLAH, M. et al. Adsorption of methylene blue on low-cost adsorbents: a review. Journal of Hazardous Materials, v. 177, p. 70-80, 2010

ROBINSON, T.; CHANDRAN, B.; NIGAM, P. Removal of dyes from a synthetic textile dye effluent bye biosorpition on apple pomace and wheat straw. Water Research, v. 36, p. 2824-2830, 2002

SMITH, K.M. et al. Sewage sludge-based adsorbents: A review of their production, properties and use in water treatment applications: review. Water Research, v. 43, p. 2569-2594, 2009.

SUN, G.; XINGJING, X. Sunflower Stalks as Adsorbents for Color Removal from Textile Wastewater. Industrial \& Engineering Chemistry Research, v. 36, p. 808-812, 1997

ULSON DE SOUZA, A. A. et al [Internet] Composto adsorvente, processo para sua preparação e processo para tratamento de efluentes. Patente INPI - PI 0703182-3, 2007 [citado 2011 Ago. 12]. Disponível em: http:// $\checkmark 3$.espacenet.com/publicationDetails/originalDocument? $C C=B R \& N R=$ $P I 0703182 A 2 \& K C=A 2 \& F T=D \&$ date $=20090428 \& D B=E P O D O C \& /$ cale $=e n E P$

WANG, X.; ZHU, N.; YIN, B. Preparation of sludge-based activated carbon and its application in dye wastewater treatment. Journal of Hazardous Materials, v. 153, p. 22-27, 2008.

WU, F.C.; TSENG, R L.; JUANG, R.S. Kinetic modeling of liquid-phase adsorption of reactive dyes and metal ions on chitosan, Water Research, v. 35, n. 3, p. 613-618, 2001 